\title{
In Situ Thermomechanical Loading for TEM Studies of Nanocrystalline Alloys
}

Thomas Koenig ${ }^{1}$, Hongyu Wang ${ }^{2}$, Kayla Cole-Piepke ${ }^{1}$, Alicia Koenig ${ }^{3}$, Sourav Garg ${ }^{1}$, Garritt Tucker ${ }^{4}$, Patrick Kung ${ }^{1}$, Tim Mewes ${ }^{3}$, Claudia Mewes ${ }^{1}$, John Nogan ${ }^{5}$, Yong Zhu ${ }^{2}$ and Gregory Thompson ${ }^{3}$

${ }^{1}$ University of Alabama, United States, ${ }^{2}$ North Carolina State University, United States, ${ }^{3}$ The University of Alabama, United States, ${ }^{4}$ Colorado School of Mines, United States, ${ }^{5}$ Sandia National Laboratories, United States

In situ microscopy methods enable the qualitative and quantitative assessment of a material's nano/microstructure evolution under different stimuli. These stimuli include annealing, mechanical loading, electrical bias, gas reactions and even electro-chemical reactions in a liquid environment. To achieve these conditions, many of these in situ devices leverage microelectromechanical systems (MEMS) to provide the stimuli [1]. In this work, the development and assessment of an in situ thermomechanical loading chip for use in transmission electron microscopy (TEM) is presented.

In this work, we replicated a MEMS chip design based on the comb-drive based system devised by Zhu [2] to address nanocrystalline stability. This chip, like the one similarly reported by Pierron [3], uses an electro-static drive system and is shown in Fig. 1(a). Here, an electrical voltage is applied across the combs which provides an electrostatic force between each comb-line, Fig. 1(b). As the voltage is increased, the combs close together resulting in further separation at the specimen mount position, or shuttle, shown in Fig. 1(c). The extent of the force applied is given by:

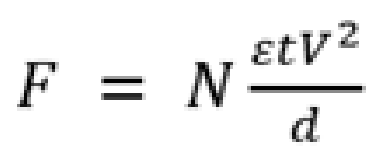

where $N$ is the number of comb pairs, $\varepsilon$ the permittivity, $V$ the applied voltage, $d$ is the inter-comb spacing, and $t$ is the active device thickness. In the initial work by Zhu and Espinosa, polycrystalline Si beams were used to provide displacement-based control through thermal expansion [4] or load-based control using the electro-static drive system [5]. In these former designs, the mechanical tests were limited to the ambient temperature. In the more recent design, electrical feed lines connected to shuttle enabled Joule heating of the specimen while under mechanical load [2]. To date, this thermomechanical loading device has been used to study a variety of nanowires such as Ag and Si nanowires in both the SEM and TEM [6-8].

To construct this MEMS device, a silicon-on-insulator (SOI) wafer is processed through a series of deposition and etch steps that yield the micro-scale comb features for a series of individual devices as seen in Fig. 1(a). These wafers consist of a handling wafer $(\sim 300-400 \mu \mathrm{m} \mathrm{SC}-\mathrm{Si})$, insulating barrier, often referred to as a buried oxide $(\mathrm{BOx})$ layer, $\left(\sim 1-2 \mu \mathrm{m} \mathrm{SiO}_{2}\right)$, and active device layer $(\sim 10-25 \mu \mathrm{m}$ SC-Si) bonded together. $\mathrm{Cr} / \mathrm{Au}$ electrical leads are then deposited atop the active device layer via e-beam deposition through a photolithographically prepared photoresist layer. Subsequently, the active comb structure is defined from the wafer through silicon deep reactive ion etching utilizing the Bosch process. 
To create the gap under the shuttle (i.e. a hole), a window is created through a two-step etch. The first is another Bosch etch that penetrates the handling wafer and a second HF etch to remove the BOx layer, which liberates comb device and shuttle to actuate freely over the window region. Finally, each individual device on the wafer must be separated from the wafer and this is achieved through laser dicing. Note that each of these steps must be precisely tuned to each device to yield a straight sidewall profile despite any significant variance in feature aspect ratios created during the above etching sequences. Failure to properly adjust the process parameters results in significant variance in the cross-sectional thickness of the MEMS chip features, resulting in inconsistent intercomb spacing and unreliable force predictions.

Here, our individual devices, measured $3 \mathrm{~mm}$ x $9 \mathrm{~mm}$, are bonded into a carrier compatible with the Hummingbird ${ }^{\circledR}$ Electrical Biasing TEM holder. These carriers consist of a shallow basin for the bonded device to reside in, a set of electrical leads, and a hole to allow for electron transmission in the specimen viewing area. The device is electrically connected to the holder probes using a wire bounding process employing Au wires, Fig. 1(d). One concern in the operation of the device can be e-beam induced drift and charging if the carrier hole is insufficiently large and/or if the device is not properly grounded on the substrate side. Fig. 2 is a plot of this effect, shown for two different spot sizes. Using a high intensity beam, one observes discontinuities at $5 \mathrm{~s}$ and $17 \mathrm{~s}$ corresponding to the discharge of the e-beam induced charge creating a significant drift. Using a low intensity beam, these discharge events did not occur and no drift was observed.

Actuation and heating are controlled remotely through source meter units (SMUs) and a custom Labview code. These SMUs provided exceptional accuracy and precision, allowing for near-infinitesimal ramping procedures of both thermal and mechanical loads on the device. The comb-drive design employed allows for actuation voltages applied to safely exceed $42.5 \mathrm{~V}(250 \mu \mathrm{N})$ without concerns for exceeding the electrical breakdown voltage in vacuum [9]. However, the device is thermally limited to $\sim 680 \mathrm{~K}$, at which the Si beams risk plastic deformation [10]. As such, ANSYS modeling is employed, informed through Raman thermometry, to predict the voltage-temperature relation within vacuum to minimize such risks.

Using this device, the thermal mechanical stability in a $\mathrm{Ni}(\mathrm{P})$ nanocrystalline alloy is investigated. These alloys use solutes to stabilize the grains from coarsening, with the solute either wetting the grain boundary or clustering to pin the boundaries. Using this in situ MEMS unit, the nanocrystalline stability is studied as a function of thermomechanical loading and grain boundary character using precession electron diffraction.

The Army Research Office grant W911NF-17-1-0528 and NASA CAN 80NSSC18M0023 supported device construction. This work was performed, in part, at the Center for Integrated Nanotechnologies, an Office of Science User Facility operated for the U.S. Department of Energy (DOE) Office of Science by Los Alamos National Laboratory (Contract 89233218CNA000001) and Sandia National Laboratories (Contract DE-NA-0003525). 

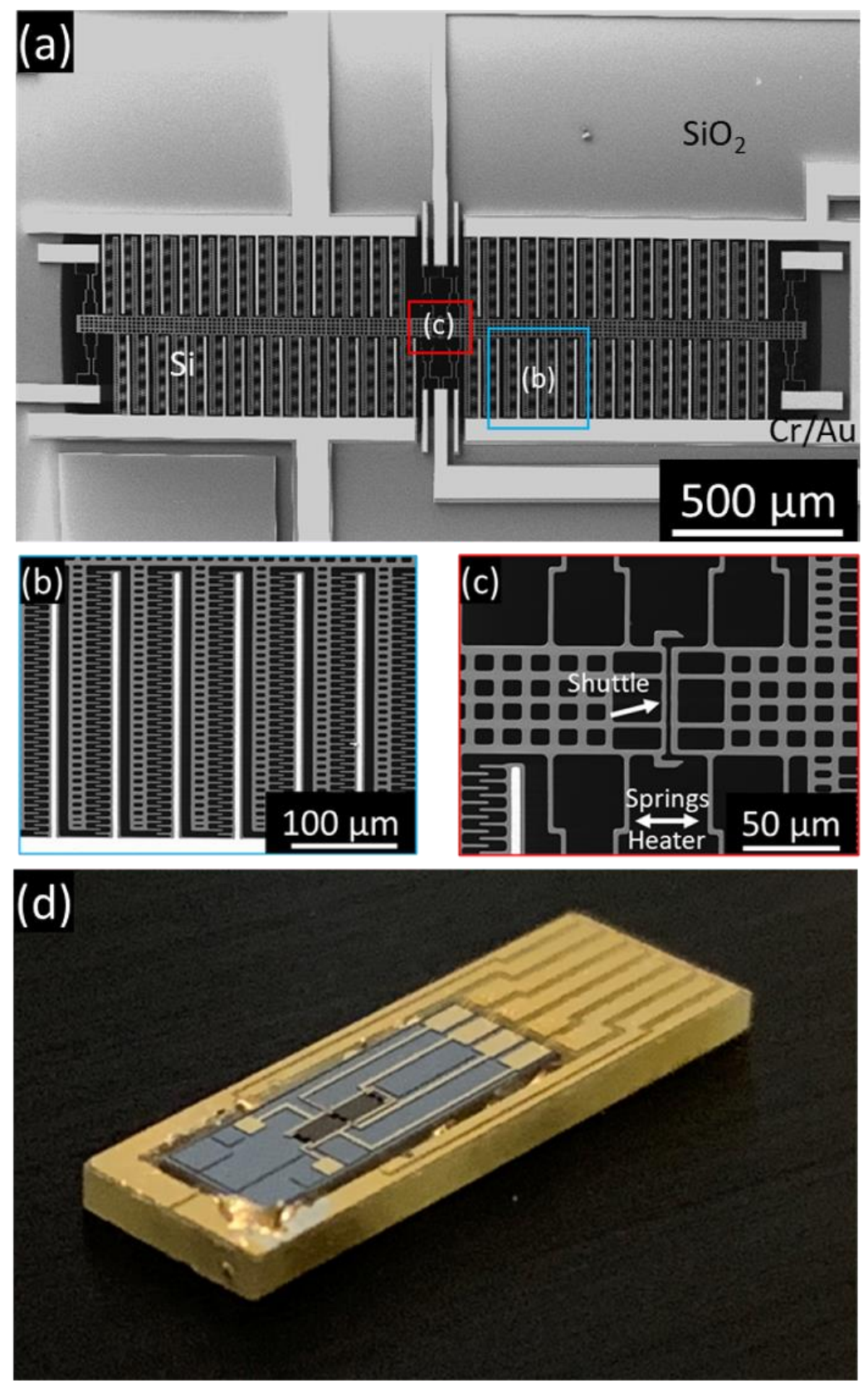

Figure 1. SEM micrographs displaying (a) an overview of the active device layer with insets (b) highlighting the comb-drive structure and (c) highlighting the specimen shuttle and dual-purpose $\mathrm{Si}$ springs/heater. Also shown in (d) is a device mounted and wire bonded into a carrier to interface to a Hummingbird $®$ electrical biasing holder. 


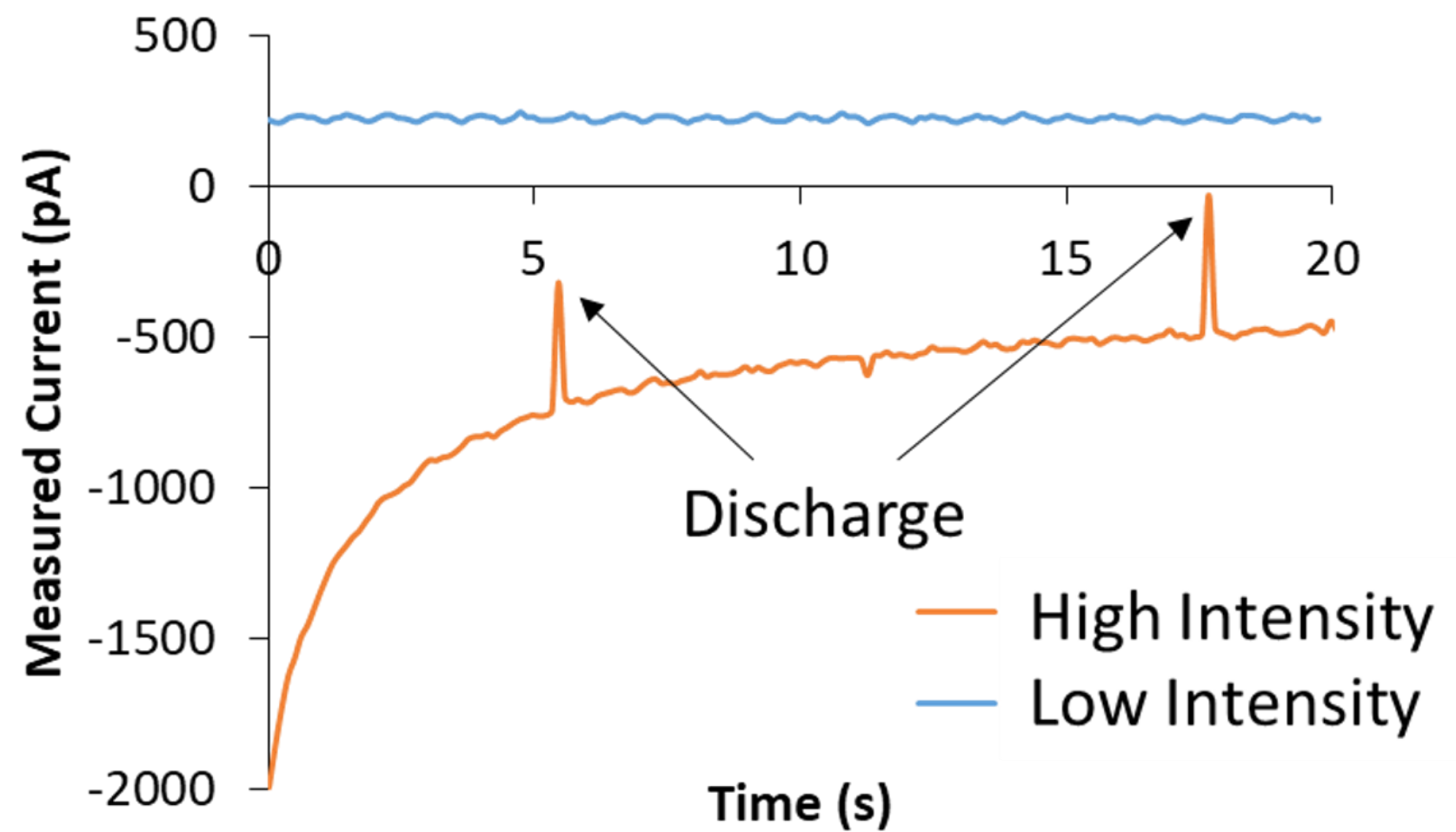

Figure 2. Measured current across the comb pair as a function of time at various e-beam intensities transmitted through the shuttle. Discharge peaks observed in the high intensity plot correspond with significant, rapid translation in the shuttle positioning. The lack of these peaks in low intensity plot also correspond with a lack of rapid translation in the shuttle positioning.

References

[1] Y. Zhu et al. A review of microelectromechanical systems for nanoscale mechanical characterization, J. Micromech. Microeng. 25 (2015) 093001. https://doi.org/10.1088/0960-1317/25/9/093001.

[2] T.-H. Chang et al. A microelectromechanical system for thermomechanical testing of nanostructures, Appl. Phys. Lett. 103 (2013) 263114. https://doi.org/10.1063/1.4858962.

[3] S. Gupta et al. Improved very high cycle bending fatigue behavior of Ni microbeams with Au coatings, Acta Materialia. 161 (2018) 444-455. https://doi.org/10.1016/j.actamat.2018.09.037.

[4] Y. Zhu et al. An electromechanical material testing system for in situ electron microscopy and applications, Proceedings of the National Academy of Sciences. 102 (2005) 14503-14508. https://doi.org/10.1073/pnas.0506544102.

[5] Y. Zhu, N. Moldovan et al. A microelectromechanical load sensor for in situ electron and x-ray microscopy tensile testing of nanostructures, Appl. Phys. Lett. $86 \quad$ (2005) 013506. https://doi.org/10.1063/1.1844594.

[6] G. Cheng et al. Anomalous Tensile Detwinning in Twinned Nanowires, Phys. Rev. Lett. 119 (2017) 256101. https://doi.org/10.1103/PhysRevLett.119.256101.

[7] G. Cheng et al. In-situ TEM study of dislocation interaction with twin boundary and retraction in twinned metallic nanowires, Acta Materialia. $196 \quad$ (2020) 304-312. https://doi.org/10.1016/j.actamat.2020.06.055. 
[8] G. Cheng et al. In Situ Nano-thermomechanical Experiment Reveals Brittle to Ductile Transition in Silicon Nanowires, Nano Lett. 19 (2019) 5327-5334. https://doi.org/10.1021/acs.nanolett.9b01789.

[9] C.-H. Chen et al. Electrical breakdown phenomena for devices with micron separations, J. Micromech. Microeng. 16 (2006) 1366-1373. https://doi.org/10.1088/0960-1317/16/7/034.

[10] S. Nakao et al. Mechanical properties of a micron-sized SCS film in a high-temperature environment, J. Micromech. Microeng. 16 (2006) 715-720. https://doi.org/10.1088/0960-1317/16/4/007. 